\title{
Directly Measuring an Entangled State
}

\section{Researchers have directly measured the components of a nonlocal, entangled wave function, rather than relying on indirect tomographic or reconstructive techniques.}

\section{by David J. Starling*}

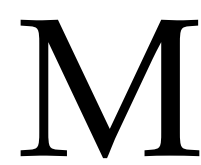

easuring the quantum state of a system is difficult. The common way, called tomography, involves measuring multiple copies of the system and using the statistics of that ensemble to algorithmically guess the closest possible quantum state [1]. A more direct method exists using so-called weak values, which are the result of weak (low-precision) measurements on a pre- and postselected quantum state [2-4]. However, the weak value approach is limited when it comes to measuring nonlocal (spatially separated) quantum states. In a new work, the group of Guang-Can Guo at the University of Science and Technology of China has shown that it is possible to directly measure the wave function of two spatially separated entangled photons [5]. Rather than weak values, the researchers employ modular values, which are character-

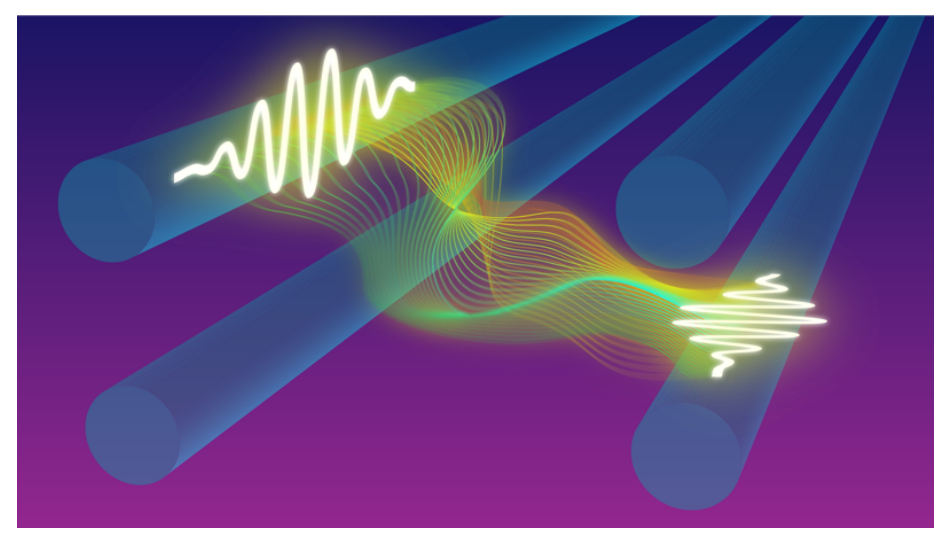

Figure 1: Researchers have directly measured the wave function of two entangled photons. The experiment generates photon pairs, which travel down four separate paths (shown in blue). The photons are entangled in such a way that measuring which paths are taken can produce a set of "modular values," which reveal the nonlocal polarization state of the photons. (APS/Alan Stonebraker)

*Division of Science, Pennsylvania State University, Hazleton, PA 18202, USA izations of a quantum system obtained by making a strong measurement of a qubit, called the meter, that is coupled to the system [6]. This demonstration may lead to more efficient methods for probing large entangled systems, as are imagined in future quantum information technologies.

To understand the context of this new work, let us consider the state of a single electron. We can think about all the possible measurements that can be made on the electron. For example, we can measure its spin direction along the $z$ axis (up or down) or along the $x$ axis. We can observe its position or its momentum in a certain direction. Quantum theory restricts these measurements by limiting the precision of knowledge obtainable for two complimentary observables, such as position and momentum. Additionally, there is the problem of measurement disturbance, where a strong measurement of one observable (position) irreversibly alters the result of another observable (momentum). However, as many have discovered, it is possible to make weak measurements where the disturbance is minimal. In these situations, some limited information can still be gained about complimentary observables without violating the strict limits set by quantum theory [7]. As an example, scientists can measure the spin of an electron by deflecting it with a magnetic field. If the magnetic field is weak enough, spin-up and spin-down electrons will only be displaced by a small amount from their mean trajectories. Such a weak measurement can leave the original spin state of the electron intact.

To extract the most from a weak measurement, researchers have devised experiments where they preselect and postselect which quantum states they look at. The output of such a measurement is called a weak value [4]. The exact meaning of the weak value has been debated, with some researchers claiming that it gives a direct view of underlying quantum features and others suggesting that weak values can violate standard quantum limits. Still, it is clear that the study of weak values represents a bone fide advance in our understanding of quantum measurement, particularly in the direct measurement of wave functions.

The first direct measurement of a wave function with weak values occurred in 2011 when Jeff Lundeen and collaborators at the Institute for National Measurement Standards in Canada recovered the spatial wave function of an en- 
semble of single photons [2]. Although their experimental technique was still statistical, in that many identical copies of the photon were measured in succession, their method bypassed the standard tomographic reconstruction to directly measure the complex amplitudes of the photon's quantum wave function [1]. Lundeen's group followed these experiments with weak measurements of a two-state system-in this case, the two orthogonal polarization states of a photon [3] (see 12 September 2016 Viewpoint). That work showed that weak values can be used to directly measure the density matrix, which is a generalization of the wave function that can account for statistically mixed ensembles of pure states.

The next step in this development would presumably be to directly measure the wave function of a two-state system, in which the individual states are entangled and spatially separated. However, the standard weak value scheme cannot be applied to nonlocal states, as it requires information to instantaneously pass between the particles during the weak measurements [6]. Researchers have found a way to obtain the weak values for nonlocal states [8], but the method requires multiple (low-probability) weak measurements. The information obtained from such measurements is very limited, which means the experiments have to be repeated a large number of times and can be impractical for entangled photon experiments.

Guo's team overcame the difficulties with weak values by switching to modular values, which also involve preand postselection of quantum states but are based on strong rather than weak measurements. The team first generated entangled photons using an ultraviolet (UV) laser and a beta barium borate (BBO) crystal that converted UV photons into lower-energy photon pairs. The photons could travel down one of four separate paths. Because of momentum conservation and the properties of the BBO crystal, the two photons in each pair were entangled in both their paths and in their polarization (Fig. 1). The team used the path state of the photons as the qubit meter in order to determine the polarization state of the entangled photons. Specifically, they postselected polarization states using wave plates and polarizing beam splitters in each path, and they used interference effects and single-photon detectors to strongly measure the paths of the photons. By analyzing the data, they could calculate the entangled polarization state. The team verified their results using standard tomographic techniques.

The measurement and characterization of entangled states is extremely important for quantum information applications such as cryptography, communication, and computing. As state-of-the-art research efforts continue to produce larger entangled quantum systems, methods to characterize those systems must similarly improve. Standard tomographic methods become difficult as system sizes increase, in part due to the requirement of global reconstruction of the full quantum state. Therefore, direct measurement methods-that scale reasonably - may provide a good option for entangled state characterization. I believe the work by Guo's group could provide such an option. It will be interesting to see if their modular value method can be expanded to atomic or superconducting systems, which are widely being studied in quantum computing applications.

This research is published in Physical Review Letters.

\section{REFERENCES}

[1] D. F. V. James, P. G. Kwiat, W. J. Munro, and A. G. White, "Measurement of qubits," Phys. Rev. A 64, 052312 (2001).

[2] J. S. Lundeen, B. Sutherland, A. Patel, C. Stewart, and C. Bamber, "Direct measurement of the quantum wavefunction," Nature 474, 188 (2011).

[3] G. S. Thekkadath, L. Giner, Y. Chalich, M. J. Horton, J. Banker, and J. S. Lundeen, "Direct measurement of the density matrix of a quantum system," Phys. Rev. Lett. 117, 120401 (2016).

[4] Y. Aharonov, D. Z. Albert, and L. Vaidman, "How the result of a measurement of a component of the spin of a spin-1/2 particle can turn out to be 100," Phys. Rev. Lett. 60, 1351 (1988).

[5] W.-W. Pan et al., "Direct measurement of a nonlocal entangled quantum state," Phys. Rev. Lett. 123, 150402 (2019).

[6] Y. Kedem and L. Vaidman, "Modular values and weak values of quantum observables," Phys. Rev. Lett. 105, 230401 (2010).

[7] M. Ozawa, "Universally valid reformulation of the Heisenberg uncertainty principle on noise and disturbance in measurement," Phys. Rev. A 67, 042105 (2003).

[8] J. S. Lundeen and K. J. Resch, "Practical measurement of joint weak values and their connection to the annihilation operator," Phys. Lett. A 334, 337 (2005).

10.1103/Physics. 12.110 